Pacific Journal of Mathematic 


\title{
SPECTRAL PROPERTIES OF LOCALLY HOLOMORPHIC VECTOR-VALUED FUNCTIONS
}

\author{
HARM BART
}

\begin{abstract}
This paper deals with spectral properties of commutative locally holomorphic Banach algebra valued functions. One of the main concepts is that of a spectral set of such a function. This concept, which is due to $L$. Mittenthal, extends that of a spectral set of a single Banach algebra element. It will be shown that the spectral idempotent associated with a nonvoid spectral set is nonzero. This result is a generalization of a well-known theorem in ordinary spectral theory. It will be used to prove a correctly stated but incorrectly proven theorem of L. Mittenthal.
\end{abstract}

We investigate spectral properties of a commutative locally holomorphic function $F$ defined on an open subset of the complex plane and with values in a complex Banach algebra $B$. In particular we will be dealing with two concepts which were introduced by $L$. Mittenthal in his dissertation [4] (see also [5]).

The first concept is that of a spectral set (i.e., a separating singular subset in terms of [4] and [5]) of $F$. We will show (Theorem 4) that the spectral idempotent associated with $F$ and a (nonvoid) spectral set of $F$ is nonzero. This result, which extends a well-known theorem in ordinary spectral theory (see [3], §5.6), seems to be new.

The second concept is that of the spectral resultant (i.e., the root operator in terms of [4] and [5]) of $F$ and a spectral set $S$ of $F$. This resultant $r$ is an element of the Banach algebra $p B p$. Here $p$ denotes the spectral idempotent associated with $F$ and $S$. Our second main result (Theorem 7) shows that $S$ is precisely the spectrum of $r$ relative to $p B p$. This also extends a well-known result in ordinary spectral theory (see [3], §5.6). Further, we will prove (Theorem 9) a generalization of the spectral mapping theorem (see [3], §5.3).

For the case when $B$ is the Banach algebra of all bounded linear operators on a complex Banach space, Mittenthal has results similar to those mentioned in the preceding paragraph (see [4], Theorems 2-4 and 2-6, and [5], Theorem 9 and Corollary 10). However, his proofs do not seem to be quite correct. In our argument, Theorem 4 , cited above, plays a crucial role.

1. Preliminaries. In this section we present some definitions and notations. The symbol $\boldsymbol{C}$ denotes the complex plane. The clo- 
sure of a subset $V$ of $C$ is denoted by $\bar{V}$. We shall often use the concept of a Cauchy domain. For the definition of this notion, we refer to [6], §5.6. The (positively oriented) boundary of a Cauchy domain $D$ is denoted by $\partial D$.

The domain of a function $f$ will be denoted by $\Delta(f)$. A Banach algebra valued function $g$ is said to be commutative if

$$
g(\lambda) g(\mu)=g(\mu) g(\lambda) \quad(\lambda, \mu \in \Delta(g)) .
$$

We shall freely use the standard notions concerning locally holomorphic vector-valued functions. For a fairly complete survey of these notions we refer to [2], §III.14.

Let $F$ be a locally holomorphic function defined on an open subset $\Delta$ of $C$ and with values in a complex Banach algebra $B$ with unit element $e$. We do not require the norm of $e$ to be one (cf. [3], $\S 1.15)$.

The set $R(F)$ of all $\lambda \in \Delta$ such that $F(\lambda)$ is regular in $B$ is called the resolvent set of $F$. It is an open subset of $C$. The function $F^{-1}$ defined by

$$
F^{-1}(\lambda)=F(\lambda)^{-1} \quad(\lambda \in R(F))
$$

is called the resolvent of $F$. It is a locally holomorphic function with values in $B$. The set $S(F)$ of all $\lambda \in \Delta$ such that $F(\lambda)$ is singular in $B$ is called the spectrum of $F$. Observe that

$$
S(F)=\Delta \backslash R(F),
$$

and that $R(F)$ is closed in the relative topology of $\Delta$.

By $Q_{F}$ we denote the function given by

$$
Q_{F}(\lambda, \mu)= \begin{cases}\frac{F(\lambda)-F(\mu)}{\lambda-\mu} & (\lambda, \mu \in \Delta ; \lambda \neq \mu), \\ F^{\prime}(\lambda) & (\lambda=\mu \in \Delta) .\end{cases}
$$

Here $F^{\prime}$ denotes, as usually, the derivative of $F$. A subset $S$ of $S(F)$ is called a spectral set of $F$ if the following three conditions are satisfied:

(i) $S$ is both open and closed in the relative topology of $S(F)$;

(ii) $S$ is a nonvoid compact subset of $C$;

(iii) $Q_{F}(\lambda, \mu)$ is regular for all $\lambda, \mu \in S$.

This notion corresponds with Mittenthal's concept of a separating singular subset.

By way of illustration, we consider the special case when

$$
F(\lambda)=\lambda e-t \quad(\lambda \in \mathcal{C}),
$$

where $t$ is some element of $B$. Then 


$$
S(F)=\sigma(t), \quad R(F)=\rho(t), \quad F^{-1}=R(\cdot ; t) .
$$

Here $\sigma(t), \rho(t)$, and $R(\cdot ; t)$ denote, as usually, the spectrum, resolvent set and resolvent of $t$ (cf. [3], Definition 4.7.1). Further, the spectral sets of $F$ are precisely the spectral sets of $t$ (cf. [3], Definition 5.6.1). This justifies our terminology.

2. Spectral idempotents. In the following $S$ denotes a spectral set of a commutative locally holomorphic function $F$ defined on an open subset $\Delta$ of $C$ and with values in a complex Banach algebra $B$ with unit element $e$. Using methods of Mittenthal, we shall introduce an "operational calculus". Further we shall define the spectral idempotent $p$ associated with $F$ and $S$. The main result of this section is that $p$ is nonzero.

Let $\mathscr{F}$ be the set of all complex-valued functions $f$ such that

(i) $\Delta(f)$ is an open neighborhood of $S$;

(ii) $f$ is locally holomorphic.

Let $\mathscr{G}$ be the set of all functions $g$ with values in $B$ such that

(i) $\Delta(g)$ is an open neighborhood of $S$;

(ii) $g$ is locally holomorphic;

(iii) $g(\lambda) F(\mu)=F(\mu) g(\lambda)$ for all $\lambda \in \Delta(g)$ and $\mu \in \Delta$.

In $\mathscr{F}$ and $\mathscr{G}$ we define algebraic operations-scalar multiplication, addition and multiplication-in an obvious way. We shall now define for each function $h$, which belongs either to $\mathscr{F}$ or to $\mathscr{G}$, an element $F_{h} \in B$ in such a way that the mappings $h \rightarrow F_{h}(h \in \mathscr{F})$ and $h \rightarrow$ $F_{h}(h \in \mathscr{G})$ preserve the algebraic operations. The definition is

$$
F_{h}=\frac{1}{2 \pi i} \int h(\lambda) F^{\prime}(\lambda) F^{-1}(\lambda) d \lambda,
$$

where $D$ is any bounded Cauchy domain such that

$$
S \subset D \subset \bar{D} \subset \Delta(h) \cap[\Delta \backslash\{S(F) \backslash S\}] .
$$

Since $\Delta(h) \cap[\Delta \backslash\{S(F) \backslash S\}]$ is an open neighborhood of the compact set $S$, there do exist bounded Cauchy domains of the required sort. It follows from Cauchy's theorem (see [2], §III.14) that the value of the above integral is independent of the choice of $D$. Thus, $F_{h}$ is well-defined (cf. [6], §5.6). The following theorem is essentially due to Mittenthal. The proof, which is similar to that of [4], Theorem 1-3 (also [5], Theorems 1 and 2), will be omitted.

Theorem 1. Let $\alpha \in \boldsymbol{C}$ and either $f, g \in \mathscr{F}$, or $f, g \in \mathscr{G}$. Then
(i) $F_{\alpha f}=\alpha F_{f}$;
(ii) $F_{f+g}=F_{f}+F_{g}$;
(iii) $F_{f g}=F_{f} F_{g}$. 
Corollary 2. Let $p \in B$ be given by

$$
p=\frac{1}{2 \pi i}{ }_{\partial D} \int F^{\prime}(\lambda) F^{-1}(\lambda) d \lambda,
$$

where $D$ is any bounded Cauchy domain such that

$$
S \subset D \subset \bar{D} \subset \Delta \backslash[S(F) \backslash S] \text {. }
$$

Then $p$ is an idempotent.

The element $p \in B$ defined in Corollary 2 plays a crucial role in this paper. It is called the spectral idempotent associated with $F$ and $S$. Suppose that $F$ is as in formula $(*)$ of $\S 1$. Then $p$ is the spectral idempotent associated with $t$ and the spectral set $S$ of $t$ (cf. [3], Theorem 5.6.1). This justifies our terminology. Furthermore, we note that, in this case, $F_{h}=p h(t)=h(t) p$ for all $h \in \mathscr{F}$. For the definition of $h(t)$ we refer to [3], Theorem 5.2.4 (see also [6], §5.6).

For the proof of the next theorem, containing the main result of this section, we need a lemma.

Lemma 3. Let $D$ be a bounded Cauchy domain such that $\bar{D} \subset \Delta$ and $\partial D \subset R(F)$. Suppose that

$$
{ }_{\partial D} F^{\prime}(\lambda) F^{-1}(\lambda) d \lambda=0 .
$$

Then $D$ is a subset of $R(F)$.

Proof. Since $F$ is commutative, the set $\{F(\lambda) \mid \lambda \in \Delta\}$ is contained in a maximal commutative subset $A$ of $B$. Observe that $A$ is a closed commutative subalgebra of $B$ with unit element $e$. An element of $A$ is regular in $A$ with inverse $y$ if and only if it is regular in $B$ with inverse $y$. Hence, without loss of generality, we may assume $B$ to be commutative.

From the Gelfand representation theory (see [3], $\S \S 4.13$ and 4.14) we know that an element $b \in B$ is regular in $B$ if and only if $\beta(b) \neq$ 0 for each (nonzero) multiplicative linear functional $\beta$ on $B$. Let $\beta$ be such a functional and put $f=\beta \circ F$. Then $f$ is a locally holomorphic complex-valued function and $f^{\prime}=\beta \circ F^{\prime}$. For $\lambda \in R(F)$ we have $f(\lambda) \neq 0$ and $f(\lambda)^{-1}=\beta\left(F^{-1}(\lambda)\right)$. It is easy to verify that

$$
{ }_{\partial D} \frac{f^{\prime}(\lambda)}{f(\lambda)} d \lambda=\beta\left(\int_{\partial D} F^{\prime}(\lambda) F^{-1}(\lambda) d \lambda\right)=\beta(0)=0 .
$$

By a well-known result from complex analysis (see [1], Ch. III, $\S 4$, Satz 16), this implies that $\beta(F(\lambda))=f(\lambda) \neq 0$ for all $\lambda \in D$, and the proof is complete. 
THEOREM 4. The spectral idempotent $p$ associated with $F$ and $S$ is nonzero.

Proof. Suppose that $p=0$. Then

$$
{ }_{\partial D} F^{\prime}(\lambda) F^{-1}(\lambda) d \lambda=2 \pi i p=0,
$$

where $D$ is as in Corollary 2. By Lemma 3, this implies that $D \subset$ $R(F)$. Consequently $S \subset R(F)$. But $S \subset S(F)$ too. It follows that $S \subset R(F) \cap S(F)=\varnothing$. This contradicts the fact that, by definition, a spectral set is nonvoid.

3. The spectral resultant. In this section we shall define the spectral resultant $r$ of $F$ and $S$. Our main result is that $r$ is an element of the complex Banach algebra $p B p$ whose spectrum (relative to $p B p$ ) is precisely $S$. Further, we shall prove a generalization of the spectral mapping theorem.

Since $p$ is a nonzero idempotent (see Theorem 4 ), $p B p$ is a closed subalgebra of $B$ with unit element $p$. The resolvent set, spectrum and resolvent of an element $x \in p B p$ relative to $p B p$ will be denoted by $\rho_{p}(x), \sigma_{p}(x)$, and $R_{p}(\cdot ; x)$ respectively. An element $x \in B$ belongs to $p B p$ if and only if $x=p x=x p(=p x p)$. As an easy consequence of Theorem 1 we have that $F_{h} \in p B p$ for each $h$ which belongs either to $\mathscr{F}$ or to $\mathscr{G}$. In particular, the element $r \in B$, given by

$$
r=\frac{1}{2 \pi i}{ }_{\partial D} \int \lambda F^{\prime}(\lambda) F^{-1}(\lambda) d \lambda
$$

where $D$ is any bounded Cauchy domain such that

$$
S \subset D \subset \bar{D} \subset \Delta \backslash[S(F) \backslash S],
$$

belongs to $p B p$. It is called the spectral resultant of $F$ and $S$. This notion corresponds with Mittenthal's concept of the root operator. If $F$ is as in formula $(*)$ of $\S 1$, then $p$ is the spectral idempotent associated with $t$ and the spectral set $S$ of $t, r=t p=p t$ and $\sigma_{p}(r)=S$ (see the proof of [3], Theorem 5.6.1). We shall prove that the last equality holds in general.

Lemma 5. Let $\mu \in C \backslash S$. Then $\mu \in \rho_{p}(r)$ and

$$
R_{p}(\mu ; r)=\frac{1}{2 \pi i}{ }_{\partial D} \int \frac{F^{\prime}(\lambda) F^{-1}(\lambda)}{\mu-\lambda} d \lambda,
$$

where $D$ is any bounded Cauchy domain such that

$$
S \subset D \subset \bar{D} \subset[C \backslash\{\mu\}] \cap[\Delta \backslash\{S(F) \backslash S\}] .
$$


Proof. The proof is similar to that of [4], Theorem 2-2 (cf. also the first part of the proof of [5], Theorem 9). Define $g: C \rightarrow C$ and $h: C \backslash\{\mu\} \rightarrow C$ by $g(\lambda)=\mu-\lambda$ and $h(\lambda)=(\mu-\lambda)^{-1}$. Clearly, both $g$ and $h$ belong to $\mathscr{F}$. By Theorem 1 , we have $F_{g} F_{h}=F_{h} F_{g}=p$ and $F_{g}=\mu p-r$. Thus $\mu p-r$ is regular in $p B p$ with inverse $F_{h}$. This proves the lemma.

Lemma 6. $\sigma_{p}(r)=\{\lambda \in S \mid p F(\lambda)$ singular in $p B p\}$.

Proof. The proof is similar to that of [4], Theorem 2-4 (cf. also the second part of the proof of [5], Theorem 9). From Lemma 5 we know that $S \subset \sigma_{p}(r)$. Therefore, it suffices to show that an element $\mu \in S$ belongs to $\sigma_{p}(r)$ if and only if $p F(\mu)$ is singular in $p B p$.

Let $\mu \in S$. Using the function $Q_{F}$, which was introduced in $\S 1$, we define the function $Q: \Delta \rightarrow B$ by $Q(\lambda)=Q_{F}(\lambda, \mu)$. It is not difficult to prove that $Q$ belongs to the set $\mathscr{G}$. Since $S$ is a spectral set of $F$, we have $S \subset R(Q)$. It follows that the resolvent $P\left(=Q^{-1}\right)$ of the function $Q$ belongs to $\mathscr{G}$ too. Applying Theorem 1, we obtain $F_{P} F_{Q}=F_{Q} F_{P}=p$. Hence $F_{Q}$ is regular in $p B p$.

Clearly, $F \in \mathscr{G}$ and $F(\mu)=F(\lambda)+(\mu-\lambda) Q(\lambda)$ for all $\lambda \in \Delta$. Using Theorem 1, we find $p F(\mu)=F_{F}+(\mu p-r) F_{Q}$. It follows from Cauchy's theorem that $F_{F}=0$. So $p F(\mu)=(\mu p-r) F_{Q}=F_{Q}(\mu p-r)$. Since $F_{Q}$ is regular in $p B p$, it follows that $\mu p-r$ is singular in $p B p$ if and only if $p F(\mu)$ is singular in $p B p$. This proves the lemma.

The next theorem contains the main result of this section. Mittenthal has a similar result (cf. [4], Theorem 2-4 and [5], Theorem 9). His proof, however, is not quite correct. In fact, Mittenthal only proved what we have called Lemma 6 . Our argument is based on Theorem 4.

\section{Theorem 7. $\sigma_{p}(r)=S$.}

Proof. In view of Lemma 6 it is sufficient to prove that $p F(\mu)$ is singular in $p B p$ for all $\mu \in S$. The case $p=e$ is trivial. Therefore, we may assume $p \neq e$.

Put $q=e-p$. Then $q$ is a nonzero idempotent and $q B q$ is a closed subalgebra of $B$ with unit element $q$. From the definition of $p$ it is clear that $F(\lambda)$ commutes with $p$ and $q$ for all $\lambda \in \Delta$. Hence $p F(\lambda) \in p B p$ and $q F(\lambda) \in q B q$ for all $\lambda \in \Delta$. By $F_{p}$ and $F_{q}$ we denote the functions, with domain $\Delta$, given by $F_{p}(\lambda)=p F(\lambda)$ and $F_{q}(\lambda)=$ $q F(\lambda)$. Observe that $F_{p}$ is a commutative locally holomorphic function with values in the complex Banach algebra $p B p$. Similarly, $F_{q}$ is a commutative locally holomorphic function with values in $q B q$. 
Let $S_{p}\left(F_{p}\right)$ denote the spectrum of $F_{p}$ (relative to $p B p$ ), and let $S_{q}\left(F_{q}\right)$ denote the spectrum of $F_{q}$ (relative to $q B q$ ). We have to prove that $S \subset S_{p}\left(F_{p}\right)$. Since $S \subset S(F)=S_{p}\left(F_{p}\right) \cup S_{q}\left(F_{q}\right)$, it suffices to show that $S \cap S_{q}\left(F_{q}\right)=\varnothing$.

Put $S_{q}=S \cap S_{q}\left(F_{q}\right)$, and suppose that $S_{q}$ is nonvoid. Then $S_{q}$ is a spectral set of $F_{q}$. The spectral idempotent associated with $F_{q}$ and $S_{q}$ is equal to $q p=0$. This contradicts Theorem 4, and the proof is complete.

Let $h \in \mathscr{F}$. The preceding theorem shows that $\sigma_{p}(r)=S$. Hence $\sigma_{p}(r) \subset \Delta(h)$. We use the symbol $h(r)_{p}$ to denote the element of $p B p$ given by

$$
h(r)_{p}=\frac{1}{2 \pi i \quad} \int h(\mu) R_{p}(\mu ; r) d \mu,
$$

where $D$ is any bounded Cauchy domain such that

$$
\sigma_{p}(r)=S \subset D \subset \bar{D} \subset \Delta(h) .
$$

From ordinary operational calculus we know that $h(r)_{p}$ is well-defined (cf. [3], Theorem 5.2.4 and [6], §5.6). It will be shown that $h(r)_{p}=$ $F_{h}$. A similar result appears in the work of Mittenthal (see [4], pp. 42, 43, 49 and [5], pp. 126-129), but again his arguments are not quite satisfactory. We shall give a new proof.

LEMma 8. $h(r)_{p}=F_{h}(h \in \mathscr{F})$.

Proof. Let $h \in \mathscr{F}$. Choose two bounded Cauchy domains $U$ and $V$ such that

$$
S \subset U \subset \bar{U} \subset V \subset \bar{V} \subset \Delta(h) \cap[\Delta \backslash\{S(F) \backslash S\}] .
$$

Then

$$
h(r)_{p}=\frac{1}{2 \pi i}{ }_{\partial V} \int h(\mu) R_{p}(\mu ; r) d \mu .
$$

By Lemma 5

$$
R_{p}(\mu ; r)=\frac{1}{2 \pi i}{ }_{\partial U} \int \frac{F^{\prime}(\lambda) F^{-1}(\lambda)}{\mu-\lambda} d \lambda
$$

for all $\mu \in \partial V$. Hence

$$
h(r)_{p}=\left(\frac{1}{2 \pi i}\right)_{\partial V}^{2} \int\left[{ }_{\partial U} \int \frac{h(\mu)}{\mu-\lambda} F^{\prime}(\lambda) F^{-1}(\lambda) d \lambda\right] d \mu .
$$

By changing the order of integration, we find 


$$
h(r)_{p}=\left(\frac{1}{2 \pi i}\right)_{\partial U}^{2} \int\left[{ }_{\partial V} \int \frac{h(\mu)}{\mu-\lambda} d \mu\right] F^{\prime}(\lambda) F^{-1}(\lambda) d \lambda .
$$

Cauchy's integral formula yields that

$$
h(\lambda)=\frac{1}{2 \pi i} \int_{\partial V} \int \frac{h(\mu)}{\mu-\lambda} d \mu
$$

for all $\lambda \in \partial U$. Thus

$$
h(r)_{p}=\frac{1}{2 \pi i} \partial h(\lambda) F^{\prime \prime}(\lambda) F^{-1}(\lambda) d \lambda .
$$

By definition, the right hand side of this equation is equal to $F_{h}$, and so the proof is complete.

Combining Theorem 7, Lemma 8 and the spectral mapping theorem, we obtain the following result (cf. [4], Theorem 2-6 and [5], Corollary 10).

THEOREM 9. $\sigma_{p}\left(F_{h}\right)=h[S](h \in \mathscr{F})$.

Proof. From Lemma 8 we know that $F_{h}=h(r)_{p}$. The spectral mapping theorem (see [3], Theorem 5.3.1) yields that $\sigma_{p}\left(h(r)_{p}\right)=$ $h\left[\sigma_{p}(r)\right]$. Now the desired result is immediate from Theorem 7, which says that $\sigma_{p}(r)=S$.

The preceding result may be viewed as a generalization of the spectral mapping theorem. To see this, take $F$ as in formula (*) of $\S 1$ and $S=\sigma(t)$.

Let $L$ be the logarithmic derivative of $F$. Thus $L$ is the function defined on $R(F)$ by

$$
L(\lambda)=F^{\prime}(\lambda) F^{-1}(\lambda) .
$$

In view of the preceding results (in particular Theorem 1), the question arises whether $L$ satisfies the resolvent equation. The following example shows that, in general, the answer is negative.

ExAmple 10. Let $t$ be a nilpotent element of $B$ of order of nilpotence 2. Define $F$ on $C$ by

$$
F(\lambda)=\lambda e+\lambda^{2} t
$$

Then $F$ is holomorphic and commutative. Using the fact that $t^{2}=$ 0 , one easily shows that $S(F)=\{0\}$ and

$$
F^{-1}(\lambda)=\frac{1}{\lambda} e-t \quad(\lambda \neq 0) .
$$

Since $F^{\prime \prime}(0)=e$ is regular, we have that $\{0\}$ is a spectral set of $F$. 
Now assume that the logarithmic derivative $L$ of $F$ satisfies the resolvent equation on a deleted neighborhood $U$ of 0 . Thus

$$
L(\lambda)-L(\mu)=(\mu-\lambda) L(\lambda) L(\mu) \quad(\lambda, \mu \in U) .
$$

Using the expression for $F^{-1}(\lambda)$ obtained above, it is easily seen that

$$
L(\lambda)=\frac{1}{\lambda} e+t \quad(\lambda \neq 0)
$$

Substituting this in the resolvent equation, we get

$$
\left(\frac{1}{\lambda} e+t\right)-\left(\frac{1}{\mu} e+t\right)=(\mu-\lambda)\left(\frac{1}{\lambda} e+t\right)\left(\frac{1}{\mu} e+t\right) \quad(\lambda, \mu \in U) .
$$

It follows by a straightforward computation that

$$
\left(\lambda^{2}-\mu^{2}\right) t=0 \quad(\lambda, \mu \in U) .
$$

But this implies that $t=0$, which contradicts the hypothesis that the order of nilpotence of $t$ is 2 . The conclusion is that $L$ does not satisfy the resolvent equation.

\section{REFERENCES}

1. H. C. H. Behnke and F. Sommer, Theorie der analytischen Funktionen einer komplexen Veränderlichen, Springer-Verlag, Berlin, 1962.

2. N. Dunford and J. T. Schwartz, Linear Operators Part I, Interscience, New York, 1967.

3. E. Hille and R. S. Phillips, Functional Analysis and Semi-groups, Amer. Math. Soc. Coll. Publ. XXXI, Providence, 1957.

4. L. Mittenthal, Operator-valued polynomials in a complex variable, and generalizations of spectral theory, Ph. D. Dissertation, University of California, Los Angeles, 1966.

5. - Operator valued analytic functions and generalizations of spectral theory, Pacific J. Math., 24 (1968), 119-132.

6. A. E. Taylor, Introduction to Functional Analysis, Wiley and Sons, New York, 1967.

Received December 1, 1972.

Wiskundig Seminarium, VRIJe Universiteit

De Boelelaan 1081, Amsterdam 11 



\section{PACIFIC JOURNAL OF MATHEMATICS}

EDITORS

RICHARD ARens (Managing Editor)

University of California

Los Angeles, California 90024

\section{R. A. Beaumont}

University of Washington

Seattle. Washington 98105

\section{J. DugundjI}

Department of Mathematics University of Southern California Los Angeles, California 90007

D. Gilbarg and J. Milgram

Stanford University

Stanford. California 94305

\section{ASSOCIATE EDITORS}
E. F. BECKENBACH
B. H. NEUMANN
F. WOLF
K. Yoshida

\section{SUPPORTING INSTITUTIONS}

\author{
UNIVERSITY OF BRITISH COLUMBIA \\ CALIFORNIA INSTITUTE OF TECHNOLOGY \\ UNIVERSITY OF CALIFORNIA \\ MONTANA STATE UNIVERSITY \\ UNIVERSITY OF NEVADA \\ NEW MEXICO STATE UNIVERSITY \\ OREGON STATE UNIVERSITY \\ UNIVERSITY OF OREGON \\ OSAKA UNIVERSITY
}

\author{
UNIVERSITY OF SOUTHERN CALIFORNIA \\ STANFORD UNIVERSITY \\ UNIVERSITY OF TOKYO \\ UNIVERSITY OF UTAH \\ WASHINGTON STATE UNIVERSITY \\ UNIVERSITY OF WASHINGTON

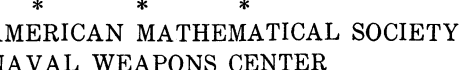

The Supporting Institutions listed above contribute to the cost of publication of this Journal, but they are not owners or publishers and have no responsibility for its content or policies.

Mathematical papers intended for publication in the Pacific Journal of Mathematics should be in typed form or offset-reproduced, (not dittoed), double spaced with large margins. Underline Greek letters in red, German in green, and script in blue. The first paragraph or two must be capable of being used separately as a synopsis of the entire paper. Items of the bibliography should not be cited there unless absolutely necessary, in which case they must be identified by author and Journal, rather than by item number. Manuscripts, in duplicate if possible, may be sent to any one of the four editors. Please classify according to the scheme of Math. Rev. Index to Vol. 39. All other communications to the editors should be addressed to the managing editor, or Elaine Barth, University of California, Los Angeles, California, 90024.

100 reprints are provided free for each article, only if page charges have been substantially paid Additional copies may be obtained at cost in multiples of 50 .

The Pacific of Journal Mathematics is issued monthly as of January 1966. Regular subscription rate: $\$ 72.00$ a year (6 Vols., 12 issues). Special rate: $\$ 36.00$ a year to individual members of supporting institutions.

Subscriptions, orders for back numbers, and changes of address should be sent to Pacific Journal of Mathematics, 103 Highland Boulevard, Berkeley, California, 94708.

\section{PUBLISHED BY PACIFIC JOURNAL OF MATHEMATICS, A NON-PROFIT CORPORATION}

Printed at Kokusai Bunken Insatsusha (International Academic Printing Co., Ltd.), 270, 3-chome Totsuka-cho. Shinjuku-ku, Tokyo 160. Japan.

Copyright (C) 1973 by Pacific Journal of Mathematics Manufactured and first issued in Japan 


\section{Pacific Journal of Mathematics}

\section{Vol. 52, No. $2 \quad$ February, 1974}

Harm Bart, Spectral properties of locally holomorphic vector-valued functions .....

J. Adrian (John) Bondy and Robert Louis Hemminger, Reconstructing infinite

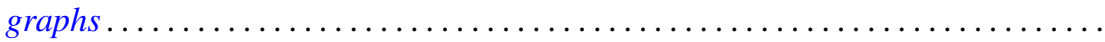

Bryan Edmund Cain and Richard J. Tondra, Biholomorphic approximation of planar

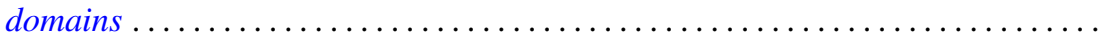

Richard Carey and Joel David Pincus, Eigenvalues of seminormal operators,

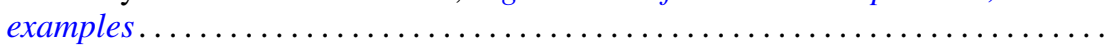

Tyrone Duncan, Absolute continuity for abstract Wiener spaces . . . . . . . . . . . . Joe Wayne Fisher and Louis Halle Rowen, An embedding of semiprime

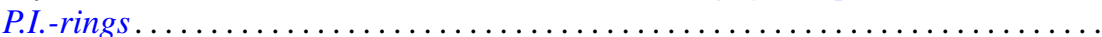

Andrew S. Geue, Precompact and collectively semi-precompact sets of semi-precompact continuous linear operators. . . . . . . . . . . . . . . .

Charles Lemuel Hagopian, Locally homeomorphic $\lambda$ connected plane continua ..... . Darald Joe Hartfiel, A study of convex sets of stochastic matrices induced by

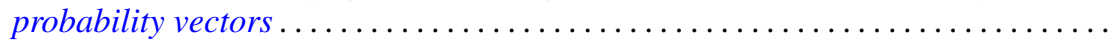

Yasunori Ishibashi, Some remarks on high order derivations $\ldots \ldots \ldots \ldots \ldots \ldots \ldots$ Donald Gordon James, Orthogonal groups of dyadic unimodular quadratic forms.

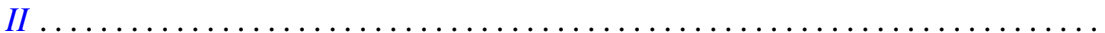

Geoffrey Thomas Jones, Projective pseudo-complemented semilattices . . . . . . . . . Darrell Conley Kent, Kelly Denis McKennon, G. Richardson and M. Schroder,

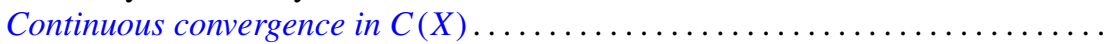

J. J. Koliha, Some convergence theorems in Banach algebras ...

Tsang Hai Kuo, Projections in the spaces of bounded linear oper

George Berry Leeman, Jr., A local estimate for typically real functions . .

475

Andrew Guy Markoe, A characterization of normal analytic spaces by the

homological codimension of the structure sheaf .........

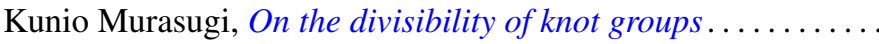

John Phillips, Perturbations of type I von Neumann algebras.

Billy E. Rhoades, Commutants of some quasi-Hausdorff matrices . .

David W. Roeder, Category theory applied to Pontryagin duality

Maxwell Alexander Rosenlicht, The nonminimality of the differential closure .

Peter Michael Rosenthal, On an inversion theorem for the general Mehler-Fock transform pair.

Alan Saleski, Stopping times for Bernoulli automorphisms

John Herman Scheuneman, Fundamental groups of compact complete locally affine complex surfaces. II. ........................

Vashishtha Narayan Singh, Reproducing kernels and operators with a cyclic vector. I. .

Peggy Strait, On the maximum and minimum of partial sums of random variables.

J. L. Brenner, Maximal ideals in the near ring of polynomials modulo 2 .

Ernst Gabor Straus, Remark on the preceding paper: "Ideals in near rings of polynomials over a field" ..........................

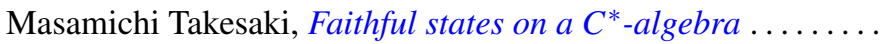

R. Michael Tanner, Some content maximizing properties of the regular simplex.

Andrew Bao-hwa Wang, An analogue of the Paley-Wiener theorem for certain

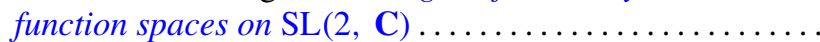

